\title{
SYSTEM THEORY BASED MULTIPLE BEAMFORMING
}

\author{
Nguyen Huu Trung*, Nguyen Thuy Anh, Nguyen Minh Duc, Doan Thanh Binh, \\ Le Trung Tan
}

\author{
Hanoi Uiversity of Science and Technology, 1 Dai Co Viet, Hanoi \\ *Email: trung.nguyenhuu@hust.edu.vn
}

Received: 11 January 2017; Accepted for publication: 5 September 2017

\begin{abstract}
In this paper, the problem of analysis and design of large-scale multiple beamforming system is considered by system theory approach. We consider the response of system parameters by set of objective functions in a critical condition, which is unable to access measurement data or the data size is large. The reduced-order model is built and the robust solution is found for a multiple beamforming system. The Monte Carlo simulation results show that the proposed multiple beamforming system yields significant performance against over existing methods.
\end{abstract}

Keywords: LS-MIMO-MC-CDMA, 5G, Multiple-beamforming, Min-max.

\section{INTRODUCTION}

Large-scale multiple beamforming systems and massive multiple input multiple output (MIMO) systems play important role in the problem of transmitted signal optimization, increasing efficiency while transmitting, optimal restoring signal at the receiver, the ability to transmit high speed data in multi-path environments (for example, in indoor environments) for telecommunications applications [1]; reducing interference in the global positioning applications [2]; target localization in sonar and radar [3]; optimizing the transmission of terrestrial Digital Video Broadcasting (multicast beamforming) [4]; etc.

The beamforming system consists of an array processor and array of sensors. It is basically a spatial filter which is used to guide the optimal radiation or absorb energy in the direction of arrival of the signal. The criteria used to optimize beamforming systems include maximal output signal-to-interference-plus-noise ratio (MSINR), minimal mean-square errors (MMSE) and minimal least-square errors (MLSE) [5].

The complexity caused by the mutual relationship between the signal processing stages of a multiple beamforming system increases with the number of antennas; especially when the system is working in a complex environment with pertubation and it has to compromise between the performance of the system and quality of service (QoS) [6]. And, the multiple beamforming system optimization by criteria, segmented by stages, often do not lead to global optimization results as expected; especially in critical condition, which is unable to access measurement data 
or the data size is large $[7,8]$. Therefore, it is necessary to develop a theoretical general model to design multiple beamforming systems on the basis of combined optimal processes to integrate the separate processes through multi-objective optimization.

On the basis of system theory and identification of dynamical systems we might consider the impact of the parameters in the system model on the objective functions under the perturbation and contraints of measurement data conditions $[9,10]$. But this process is a strong nonlinearity, complex in terms of real time calculation, especially in cases when the calibration is required or requires high accuracy in working domain $[9,10]$. However, for the challenges of real time due to the complex nonlinearity, linearization of model system, treatment processes (essentially model order reduction), applying robust theory to reduce the order of the model is widely recognized as the ultimate method $[11,12]$.

In this paper, two-stage analysis and design of multiple beamforming systems is approached by system theory. In the first stage, a reduced order model is built. In the next stage, optimal robustness is defined and solved by robust theory. This stage includes two tasks. The first task is to apply system theory to dynamical system such as Bounded Input-Bounded Output (BIBO) or conditions for controllability, stabilisability and observability of linear dynamical system $[13,14]$ to define set of necessary conditions of robust problem when the system is not affected by perturbation. Secondly, under perturbation, dynamical model (parameters, order and model characteristics) changes, sufficient condition is defined in order to preserve model characteristics as not being perturbed. Obviously, robust problem here determines the existence and the uniqueness of the solution (sufficient condition defines the limit of the solution domain). The problem is built on the basis of applying systems theory to model-order reduction.

Applied to the case of multiple beamforming system based on min-max criterion, a system model is proposed on the basis of model order reduction and the necessary, sufficient conditions shall be determined accordingly. Note that with a dynamical system model, there exists many different optimal reduced-order models due to different principles and criteria for model order reduction, leading to the existence of multiple sets of necessary, sufficient conditions. But, for the reduced-order models which are obtained by using the same principle, we could move from model to model, including the set conditions (necessary and sufficient) because in this case we use the same principle and reduced-order models comply parsimony principle. Moreover, if we use the same optimal principle, there always exists optimal projection between models [15]. In this paper, the reduced-order model obtained by applying state-optimal projection on the basis of an assumption model with nominal values and an optimum projection in min-max criterion multiple beamforming system. The condition set which needs to be determined provided that static users correspond with the cases where the system is not affected by diffraction is the set of limits to the nominal value of the decay model in the pre-selected model in the case the parameters unchanged.. The set of sufficient conditions include inequality that is determined with assuming that the users are moving according to the case that the system is perturbed, the multiple beamforming system works as in nominal mode. Obviously, the nature of a dynamical system theory (controllability, stabilisability and observability) is found in the implementation of optimization process.

The simulation results prove two problems. First, the proposed model is the same as theorical solution. Second, the proposed model yields significant performance against over existing methods.

The rest of the paper is organized as follows. In section II, the necessary background is given about beamforming methods. Section III presents the proposed min-max criterion multiple beamforming system. Section IV provides simulation results and characteristics of the proposed 
system. Finally, the conclusions of this paper are for concluding remarks, and suggestions for further researches.

\section{PRELIMINARIES}

\subsection{Signal model}

Consider a beamforming system with $M$ elements. Denote $s(t)$ is transmitted signal, the direction of arrival (DOA) angle of the wavefront plane associated with $s(t)$ is $\theta$, vector of array observation from $M$ elements at time instant $t$ is expressed as :

$$
\mathbf{x}(t)=\boldsymbol{a}(\theta, \omega) s(t)+\mathbf{i}(t)+\mathbf{n}(t)
$$

State equation

$$
\dot{\mathbf{x}}(t)=\mathbf{A x}(t)+\mathbf{i}(t)+\mathbf{n}(t)
$$

where $\boldsymbol{a}(\theta, \omega)$ is steering vector, $\mathbf{i}(t)$ is the interference and $\mathbf{n}(t)$ is a Gaussian noise vector.

$$
\boldsymbol{a}(\theta, \omega)=\left[\begin{array}{lll}
1 & e^{j \omega d \sin (\theta) / c} e^{j \omega 2 d \sin (\theta) / c} \ldots & e^{j \omega(M-1) d \sin (\theta) / c}
\end{array}\right]^{H}
$$

with: $d$ is the distance between the two elements, $\omega$ is the carrier frequency and $c$ is the speed of light. Steering vector depends on the direction of arrival and the frequency. For simplicity, we denote $\boldsymbol{a}(\theta, \omega)$ is $\boldsymbol{a}$. The single beamforming model is expressed as $\mathbf{x}(t)=\boldsymbol{a} s(t)+\mathbf{i}(t)+\mathbf{n}(t)$.

The multiple beamforming model is expressed as:

$$
\begin{aligned}
\mathbf{x}(t) & =\mathbf{A} s(t)+\mathbf{i}(t)+\mathbf{n}(t) \\
\dot{\mathbf{x}}(t) & =\mathbf{A x}(t)+\mathbf{i}(t)+\mathbf{n}(t)
\end{aligned}
$$

where $\mathbf{A}=\left[\boldsymbol{a}\left(\theta_{1}, \omega_{1}\right), \boldsymbol{a}\left(\theta_{2}, \omega_{2}\right), \ldots \boldsymbol{a}\left(\theta_{P}, \omega_{P}\right)\right]$ according to $P$ signal sources.

There are two general beamforming systems, including narrow band beamforming and broad band beamforming. In narrow band beamforming model, the output signal of beamformer at time instant $t$ is $y(t)$ obtained by linear combination of signals of $M$ elements as:

$$
y(t)=\sum_{i=1}^{M} w_{i}^{*} x_{i}(t)
$$

For broad band model, the output signal is expressed as:

$$
y(t)=\sum_{i=1}^{M} \sum_{p=0}^{K-1} w_{i, p}^{*} x_{i}(t-p)
$$

with $K-1$ is number of delay stages at each channel of $i^{\text {th }}$ element of the array. The output signal is expressed as:

$$
y(t)=\boldsymbol{w}^{H} \mathbf{x}(t)
$$

where $\mathbf{x}$ is the received signal vector. Vector $\mathbf{w}$ of length $M$ represents the weights as:

$$
\mathbf{w}^{H}=\left[w_{0}^{*}, w_{1}^{*}, \ldots, w_{K-1}^{*}\right]=\left[\mathbf{w}^{T}\right]^{*}
$$

The response of single beamformer is expressed as:

$$
r(\theta, \omega)=\mathbf{w}^{\boldsymbol{H}} \mathbf{a}
$$

The beampattern is defined as squared magnitude of $r(\theta, \omega)$. Note that each of weight in vector $\mathbf{w}$ impacts to the response of beamformer in terms of time and space.

Output power or variance of estimated signal is determined as:

$$
E\left\{|y|^{2}\right\}=\mathbf{w}^{H} E\left\{\mathbf{x} \mathbf{x}^{H}\right\} \mathbf{w}
$$


where $E\{$.$\} denotes mean.$

If the signal is wide sense stationary, the covariance matrix $\boldsymbol{R}_{x}=E\left\{\mathbf{x} \mathbf{x}^{H}\right\}$ is statistically independent over time. Although signal is not often stationary, but we design and evaluate the performance of optimized beamforming based on the hypothesis that the signal is wide sense stationary.

The covariance matrix of the narrow band signal $s(t)$ at frequency $\omega_{0}$ is:

$$
\mathbf{R}_{x}=\sigma_{s}^{2} \boldsymbol{a}\left(\theta, \omega_{0}\right) \boldsymbol{a}^{\boldsymbol{H}}\left(\theta, \omega_{0}\right)=\sigma_{s}^{2} \boldsymbol{a} \boldsymbol{a}^{\boldsymbol{H}}
$$

Where $\sigma_{s}^{2}$ is the average signal power.

\subsection{Statistically optimal beamforming techniques}

Beamforming is an important technique in array processing in order to optimize desired signal while minimizing interferences. Statistically optimal beamforming techniques include maximization of signal-to-noice ratio (SNR), Minimum Mean Squared Error (MMSE), Linearly Constrained Minimum Variance (LCMV) and minimum variance distortionless response (MVDR) are widely applied [5,8]. Design of the beamformer under statistically optimal method requires statistical properties of the source and the statistical characteristics of the channel.

\subsubsection{Maximization of SNR}

The weight vector is solution of maximization of SNR problem:

$$
\mathbf{w}_{\text {MaxSNR }}=\underset{\mathbf{w}}{\operatorname{argmax}} \frac{\mathbf{w}^{H} \mathbf{R}_{s} \mathbf{w}}{\mathbf{w}^{H} \mathbf{R}_{n} \mathbf{w}}
$$

General solution $\mathbf{w}_{\text {MaxSNR }}$ requires both $\mathbf{R}_{s}=E\left\{\mathbf{s} \mathbf{s}^{H}\right\}$ and $\mathbf{R}_{n}=E\left\{\mathbf{n n}^{H}\right\}$ to be of covariance matrices of signal and noise. Depending on applications, the calculations of $\mathbf{R}_{s}$ and $\mathbf{R}_{n}$ are different. For example, $\mathbf{R}_{\mathbf{n}}$ can be estimated during absence of signal, $\mathbf{R}_{\mathbf{s}}$ is estimated from signal and known DOA by equation (10). We have, multiplying the weight vector by a scale is not changing SNR. Because steering vector $\boldsymbol{a}(\theta, \omega)$ is fixed for a fixed signal, choose a weight vector to satisfy $\mathbf{w}^{H} \boldsymbol{a}(\theta, \omega)=c$ with $c$ is a constant. The problem of SNR maximization becomes minimizing interference:

$$
\mathbf{w}_{\text {MaxSNR }}=\underset{\mathbf{w}}{\operatorname{argmax}}\{S N R\}=\underset{\mathbf{w}}{\operatorname{argmin}}\left(\mathbf{w}^{H} \mathbf{R}_{n} \mathbf{w}\right), \text { s.t. } \mathbf{w}^{H} \boldsymbol{a}=c
$$

Using the method of Lagrange multipliers, solution of the equation [8]:

$$
\mathbf{w}=c \frac{\mathbf{R}_{n}^{-1} \boldsymbol{a}}{\boldsymbol{a}^{H} \mathbf{R}_{n}^{-1} \boldsymbol{a}}
$$

\subsubsection{Minimum Mean Squared Error, MMSE}

Minimum Mean Squared Error method minimizes the error signal between transmitted signal and a reference signal $d(t)$. In this model, desired user assumes to transmit this reference signal, i.e. $s(t)=\alpha d(t)$ where $\alpha$ is amplitude of reference signal $d(t)$ and $d(t)$ is known at the receiver. The output signal of the beamformer is to track reference signal [16]. MMSE method seeks the weight to minimize average error signal power:

$$
\mathbf{w}_{M M S E}=\underset{\mathbf{w}}{\operatorname{argmin}} E\left\{|e(t)|^{2}\right\}
$$

The average error signal power: 


$$
\begin{aligned}
E\left\{|e(t)|^{2}\right\} & =E\left\{\left|\mathbf{w}^{H} \mathbf{x}(t)-d(t)\right|^{2}\right\}=E\left\{\left|\mathbf{w}^{H} \mathbf{x} \mathbf{x}^{H} \mathbf{w}-\mathbf{w}^{H} \mathbf{x} d^{*}-\mathbf{x}^{H} \mathbf{w} d+d d^{*}\right|^{2}\right\} \\
& =\mathbf{w}^{H} \mathbf{R} \mathbf{w}-\mathbf{x}^{H} \mathbf{r}_{x d}-\mathbf{r}_{x d}^{H} \mathbf{w}+d d^{*}
\end{aligned}
$$

where $\mathbf{r}_{x d}=E\left\{\mathbf{x} d^{*}\right\}$

Derivative (15) by $\mathbf{w}^{H}$ and set to zero:

$$
\frac{\partial E\left\{|e(t)|^{2}\right\}}{\partial \mathbf{w}^{H}}=\mathbf{R} \mathbf{w}-\mathbf{r}_{x d}=0
$$

We have the solution:

$$
\mathbf{w}_{M M S E}=\mathbf{R}^{-1} \mathbf{r}_{x d}
$$

This solution is known as optimal Wiener filter. This method requires reference signal to train the beamformer.

\subsubsection{Linearly Constrained Minimum Variance}

The LCMV method belongs to minimization of output power of the beamformer methods. This method keeps the response according to direction of arrival of the desired signal fixed in order to preserve desired signal while minimizing the impact of undesired components including noise and interference that come from other directions other than desired direction.

We have the output response of signal source with direction of arrival $\theta$ and frequency $\omega$ is determined by $\mathbf{w}^{\boldsymbol{H}} \boldsymbol{a}(\theta, \omega)$. Linear constraint for the weighs satisfies $\mathbf{w}^{\boldsymbol{H}} \boldsymbol{a}(\theta, \omega)=c$, where $c$ is a constant to ensure that all signals with frequency $\omega$ come from direction of arrival $\theta$ are passed with response $c$. Minimization of output due to interference is equipvalent to minimizing the output power (minimum ouput power):

$$
\mathbf{w}_{M o P}=\arg \min _{\mathbf{w}} E\left\{|y|^{2}\right\}=\arg \min _{\mathbf{w}}\left\{\mathbf{w}^{\boldsymbol{H}} \mathbf{R}_{\mathbf{x}} \mathbf{w}\right\}, \text { s.t. } \mathbf{w}^{\boldsymbol{H}} \boldsymbol{a}(\theta, \omega)=c
$$

Using the method of Lagrange multipliers, find $\min _{\mathbf{w}}[\mathcal{L}(\mathbf{w} ; \lambda)]$ where:

$$
\begin{aligned}
\mathcal{L}(\mathbf{w} ; \lambda) & =E\left\{\left|\mathbf{w}^{H} \mathbf{x}\right|^{2}\right\}+\lambda\left(\mathbf{w}^{H} a-c\right)=\mathbf{w}^{H} \mathbf{R}_{\mathbf{x}} \mathbf{w}+\lambda\left(\mathbf{w}^{H} a-c\right) \\
\frac{\partial \mathcal{L}}{\partial \mathbf{w}^{H}} & =\mathbf{R}_{\mathbf{x}} \mathbf{w}+\lambda \boldsymbol{a}
\end{aligned}
$$

Solution of the equation:

$$
\mathbf{w}_{L C M V}=-\lambda \mathbf{R}_{x}^{-1} \boldsymbol{a}=c \frac{\mathbf{R}_{x}^{-1} \boldsymbol{a}}{\boldsymbol{a}^{H} \mathbf{R}_{x}^{-1} \boldsymbol{a}}
$$

In practice, uncorrelated noise component ensures $\boldsymbol{R}_{\boldsymbol{x}}$ is invertible. If $c=1$ the beamformer is called minimum variance distortionless response, MVDR, beamformer. Solution of MVDR beamformer is equipvalent to maximization of SNR solution by replacing $\sigma^{2} \boldsymbol{a}(\theta, \omega) \boldsymbol{a}^{H}(\theta, \omega)+$ $\mathbf{R}_{\boldsymbol{n}}$ by $\boldsymbol{R}_{\boldsymbol{x}}$ and applying invert matrix lemma $[\mathbf{A}+\mathbf{B C D}]^{-1}=\mathbf{A}^{-1}-\mathbf{A}^{-1} \mathbf{B}\left[\mathbf{D} \mathbf{A}^{-1} \mathbf{B}+\right.$ $\left.\mathbf{C}^{-1}\right]^{-1} \mathbf{D A}^{-1}$, we have:

$$
\begin{gathered}
\mathbf{R}_{x}^{-1}=\left[\mathbf{R}_{n}+\sigma_{s}^{2} \boldsymbol{a} \boldsymbol{a}^{\boldsymbol{H}}\right]^{-1}=\mathbf{R}_{n}^{-1}-\frac{\mathbf{R}_{n}^{-1} \boldsymbol{a} \boldsymbol{a}^{H} \mathbf{R}_{n}^{-1}}{\boldsymbol{a}^{H} \mathbf{R}_{n}^{-1} \boldsymbol{a}+\sigma_{s}^{-2}} \\
\Rightarrow \mathbf{R}_{x}^{-1} \boldsymbol{a}=\mathbf{R}_{n}^{-1} \boldsymbol{a}-\frac{\mathbf{R}_{n}^{-1} \boldsymbol{a} \boldsymbol{a}^{H} \mathbf{R}_{n}^{-1} \boldsymbol{a}}{\boldsymbol{a}^{H} \mathbf{R}_{n}^{-1} \boldsymbol{a}+\sigma_{s}^{-2}}=\mathbf{R}_{n}^{-1} \boldsymbol{a}-\frac{\left(\boldsymbol{a}^{H} \mathbf{R}_{n}^{-1} \boldsymbol{a}\right) \mathbf{R}_{n}^{-1} \boldsymbol{a}}{\boldsymbol{a}^{H} \mathbf{R}_{n}^{-1} \boldsymbol{a}+\sigma_{s}^{-2}}=\left(\frac{\sigma_{s}^{-2}}{\boldsymbol{a}^{H} \mathbf{R}_{n}^{-1} \boldsymbol{a}+\sigma_{s}^{-2}}\right) \mathbf{R}_{n}^{-1} \boldsymbol{a} \\
=c \mathbf{R}_{n}^{-1} \boldsymbol{a}
\end{gathered}
$$




\section{SYSTEM THEORY BASED MULTIPLE BEAMFORMING PROPOSAL}

This part proposes multiple beamforming model on basis of system theory and identification of dynamical systems and set of necessary and sufficient conditions is determined accordingly.

\subsection{Problem statement}

For a beamforming system that is expressed in equation (1). The presented algorithms are full rank optimal algorithms. However, full rank methods have limitation that require a large number of samples in order to archive stable state when the number of elements of the filter is large. Hence, for large scale beamforming system, full rank methods require a large number of calculations. On the other hand, when the number of elements is large, the performance of the system is bad under pertubation. Model order reduction of a dynamical system becames obvious tool to capture the initial understanding of the system and control system to meet the speed in real time [17].

\subsubsection{Linearization for a nonlinear system which is essentially order-reduction}

Given a multiple beamforming system model of order $M$, linear, time invariant and not necessarily simultaneously controllability and observability described by reduced-order nonlinear model:

$$
\begin{aligned}
& \mathbf{x}(t)=\mathbf{A} s(t)+\mathbf{i}(t)+\mathbf{n}(t) \\
& \dot{\mathbf{x}}(t)=\mathbf{A x}(t)+\mathbf{i}(t)+\mathbf{n}(t) \\
& y(t)=\boldsymbol{w}^{H} \mathbf{x}(t)
\end{aligned}
$$

where $\mathbf{x}(t), \mathbf{i}(t)$ and $\mathbf{n}(t)$ are $M$ dimension vectors, the matrix $\mathbf{A}$ is corresponding to $P$ signal sources of the appropriate dimension and matrix A decsribes dynamical characteristics of the system. Let's define a model of order $r$, called reduced-order model, $q \leq r<M$ :

$$
\begin{aligned}
& \mathbf{x}_{r}(t)=\mathbf{A}_{r} s(t)+\mathbf{i}_{r}(t)+\mathbf{n}_{r}(t) \\
& \dot{\mathbf{x}}_{r}(t)=\mathbf{A}_{r} \mathbf{x}(t)+\mathbf{i}_{r}(t)+\mathbf{n}_{r}(t) \\
& y_{r}(t)=\mathbf{w}_{r}^{H} \mathbf{x}_{r}(t)
\end{aligned}
$$

Satisfying the following conditions:

(i). $\boldsymbol{L}_{2}$ criteria for order reduction:

$$
J_{2}\left(\mathbf{w}_{r}^{H}\right)=E\left\{\left|y_{r}\right|^{2}\right\}=E\left\{\left|\mathbf{w}_{r}^{H} \mathbf{x}_{r}\right|^{2}\right\}=\mathbf{w}_{r}^{H} \overline{\mathbf{R}}_{\mathbf{x}} \mathbf{w}_{\boldsymbol{r}}
$$

Constrained by:

$$
\mathbf{w}_{r}^{H} \mathbf{A}_{r}(\theta, \omega)=c
$$

is minimized and:

(ii). Model $\left(\mathbf{w}_{r}^{H}, \mathbf{A}_{r}\right)$ has simultaneously controllability and observability.

\subsubsection{The robustness of reduced-order model}

There are many optimization methods for the problem that was stated, in this paper, the authors use the state-optimization method [17]. 
For a linear model, time invariant of degree $\mathrm{M}$ for beamforming system, there exists a transformation $\mathbf{T}_{\mathrm{r}}$ of dimension $r \mathrm{x} M$ full row rank on to model states such as optimal parameters of reduced-order model are calculated as following [10, 11]. Choose a sample reduced-order model (assumed) which is described by:

$$
\begin{aligned}
\dot{\mathbf{x}}_{m} & =\mathbf{A}_{m} \mathbf{x}_{m}+\mathbf{B}_{m} \mathbf{u}(t) \\
\mathbf{u}(t) & =\mathbf{i}(t)+\mathbf{n}(t) \\
\mathbf{y}_{m} & =\mathbf{C}_{m} \mathbf{x}_{m}
\end{aligned}
$$

Condition equation:

$$
\mathbf{F}_{m}(z)=\mathbf{C}_{m}\left(\mathbf{I}_{z}-\mathbf{A}_{m}\right) \mathbf{B}_{m}
$$

The matrix $\mathbf{A}_{m}$ determines pole position that decides stability of the system. In order to maintain the stability of the system, the matrix $\mathbf{A}_{m}$ would not create the pole outside the unit circle. The component $\mathbf{C}_{m} \mathbf{B}_{m}$ creates points in $z$ space:

$$
\begin{aligned}
& \mathbf{A}_{r}=\mathbf{T}^{H} \mathbf{A}_{m} \mathbf{T}_{r}^{-H} \\
& \mathbf{B}_{r}=\mathbf{T}_{r}^{H} \mathbf{B} \\
& \mathbf{C}_{r}=\mathbf{C}_{m} \mathbf{T}_{r}^{-H} \\
\mathbf{x}_{\mathrm{r}}(t)= & \mathbf{T}_{r}^{H} \mathbf{x}(i), \mathbf{A}_{r}=\mathbf{T}_{r}^{H} \mathbf{A}
\end{aligned}
$$

Transformation matrix $\mathbf{T}_{r}$ is defined by minimizing objective function:

$$
\begin{gathered}
J_{2}\left(\mathbf{T}_{r}, \mathbf{w}_{r}^{H}\right)=E\left\{\left|\mathbf{w}_{r}^{H} \mathbf{T}_{r}^{H} \mathbf{x}\right|^{2}\right\}=\mathbf{w}_{r}^{H} \mathbf{T}_{r}^{H} \mathbf{R}_{x} \mathbf{T}_{r} \mathbf{w}_{r} \\
\text { s.t. } \mathbf{w}_{r}^{H} \mathbf{A}_{r}(\theta, \omega)=c
\end{gathered}
$$

Modify the constrained optimization problem (40) to unconstrained problem by the method of Lagrange multipliers as:

$$
\mathcal{L}\left(\mathbf{T}_{r} ; \mathbf{w}_{r}^{H}\right)=E\left\{\left|\mathbf{w}_{r}^{H} \mathbf{T}_{r}^{H} \mathbf{x}\right|^{2}\right\}+2 \Re\left[\lambda\left(\mathbf{w}_{r}^{H} \mathbf{T}_{r}^{H} \mathbf{A}_{r}-c\right)\right]
$$

where $\lambda$ is Lagrange multiplier, $\mathbf{w}_{r}$ is fixed, minimized by $\mathbf{T}_{r}$, solved by $\lambda$, we have:

$$
\mathbf{T}_{r}=c \frac{\mathbf{R}_{x}^{-1} \mathbf{A}_{r} \mathbf{w}_{r}^{H} \overline{\mathbf{R}}_{x}^{-1}}{\mathbf{w}_{r}^{H} \overline{\mathbf{R}}_{x}^{-1} \mathbf{w}_{r} \mathbf{A}_{r}^{H} \mathbf{R}_{x}^{-1} \mathbf{A}_{r}}
$$

\subsection{Robust optimization by min-max criteria}

Problem of robust optimization finds beamforming weight solution which minimize the worst case (the best of the worst conditions) on set of signal $\mathbf{r}_{f}$ (in time domain or frequency domain for frequency beamformers) and by $\operatorname{MSE}\left(\mathbf{r}_{f}, \hat{\mathbf{r}}_{f}\right)$ criteria, with a constant $q>0$ and a positive define matrix $\mathbf{Q}$ [18]. The problem is stated in min-max optimization as:

$$
\begin{gathered}
\mathbf{w}_{M N M}=\arg \min _{\mathbf{w}_{r}} \max _{\mathbf{r}_{f}: \mathbf{r}_{f}^{H} \mathbf{Q r _ { f }} \leq q^{2}} \operatorname{MSE}\left(\mathbf{r}_{f}, \hat{\mathbf{r}}_{f}\right) \\
=\arg \min _{\mathbf{w}_{r}} \max _{\mathbf{r}_{f}: \mathbf{r}_{f}^{H} \mathbf{Q} \mathbf{r}_{f} \leq q^{2}} E\left\{\left|\hat{\mathbf{r}}_{f}-\mathbf{r}_{f}\right|^{2}\right\} \\
=\arg \min _{\mathbf{w}_{r}} \max _{\mathbf{r}_{f}: \mathbf{r}_{f}^{H} \mathbf{Q} \mathbf{r}_{f} \leq q^{2}}\left\{\mathbf{w}_{r}^{H} \overline{\mathbf{R}}_{x} \mathbf{w}_{\boldsymbol{r}}+\left|\mathbf{r}_{f}\right|^{2}\left|1-\mathbf{w}_{r}^{H} \mathbf{A}_{r}\right|^{2}\right\}
\end{gathered}
$$

where the covariance matrix of observation vector $\overline{\mathbf{R}}_{x}=E\left\{\left(\mathbf{r}_{f} \mathbf{r}_{f}^{H}\right)\right\}$.

$$
\overline{\mathbf{w}}_{M N M}=\arg \min _{\mathbf{w}_{r}} \max \underset{\substack{\mathbf{r}_{f}: \mathbf{r}_{f}^{H} \mathbf{Q r}_{f} \leq q^{2} \\ \overline{\mathbf{R}}_{x}: \sum \max \left\{\operatorname{tr}\left(\overline{\mathbf{R}}_{x} \overline{\mathbf{R}}_{x}^{H}\right)\right\}}}{\operatorname{MSE}\left(\mathbf{r}_{f}, \hat{\mathbf{r}}_{f}\right)}
$$


Solution of the problem is determined by the method of Lagrange multipliers:

$$
\overline{\mathbf{w}}_{M N M}=q^{2} \frac{\overline{\mathbf{R}}_{x}^{-1} \mathbf{A}_{r}}{1+q^{2} \mathbf{A}_{r}^{H} \overline{\mathbf{R}}_{x}^{-1} \mathbf{A}_{r}}
$$

Approximate solution and weight vector can be found by adaptive methods such as steepest descent, conjugate direction, gradient, conjugate LMS (Least Mean Squares) and interactive Least Mean Squares [19].

\section{NUMERICAL RESULTS}

\subsection{Simulation method}

The performance of the system is performed by means of the Monte-Carlo simulation. The simulation estimates the influence of some parameters on the performance of the system. These parameters include ISR (Interference to Signal Ratio); SNR, array configuration (ULA, URA and UCA); Number of antennas $(M)$; sampling rate $f_{s}$; Difference DOA between transmited signal and interference $\Delta \theta$.

Monte-Carlo simulation algorithm includes sequence steps: generation of transmitted signal, interference and AWGN by parameters of SNR, INR and DOAs; Reception of signal by steering vector $\boldsymbol{a}(t)$, interference and AWGN at sensors; Beamforming, beamforming weights calculation by processing signal samples; Compare output signal to source signal and evaluate NRMSE by Monte-Carlo method.

Transmitted signal is determined narrow band sine wave signal. Signal is transmitted continuously through the training sequence and the amplitude of the signal can vary or change in order to get the desired SNR at each antenna. The transmitted signal is of the form:

$$
s(n)=\sqrt{S N R} e^{i 2 \pi\left(f_{c} / f_{s}\right) n+\theta}
$$

where $f_{c}$ is carrier frequency, $f_{s}$ is sampling frequency, $\theta$ is phase of signal, $n=[1: N]$ with $N$ is simulated number of samples.

Interference can be narrow band with the same frequency as signal or broadband interference as:

$$
i(t)=\sqrt{\operatorname{INR}} \mathcal{N}(0,1)
$$

AWGN $n(t)=\mathcal{N}(0,1)$ has normal standard deviation 1 appears at every antennas.

The system performance in simulation is Normalized Root Mean Square Error, NRMSE, the final value is the average value of all $Q$ values after each simulation:

$$
N R M S E=\operatorname{mean}_{1 \leq q \leq Q}\left\{\frac{\sqrt{\frac{1}{N} \sum_{k=1}^{N}\left|\hat{x}_{q}(k)-x_{q}(k)\right|^{2}}}{\left|\max \left(x_{q}(k)\right)-\min \left(x_{q}(k)\right)\right|}\right\}
$$

Besides the simulation of system performance according to SNR we also simulate the perfoermance of the system according to the interference. The severity of the interference is determined by INR (interference to noise ratio):

$I S R_{[d B]}$ (interference to signal ratio) is in $\mathrm{dB}$.

\subsection{Simulation schemes and results}




\subsubsection{Signal estimation when SNR varies}

In simulation schemes A, B, C the configuration of array is ULA (uniform linear array) with number of Massive MIMO antennas is 32, the distance between two consecutive antennas is $\lambda / 2$. For GPS simulation L1 frequency is $f_{c}=1575.42 \mathrm{MHz}$ this distance is $9.52 \mathrm{~cm}$. Because this distance limits the dimension of the system. Therefore, for GPS, the configuration is UCA-7 and URA-9 (Uniform Circular Array). UCA-7 is shown in Fig. 1, URA-9 is the same, of the form $3 \times 3$.

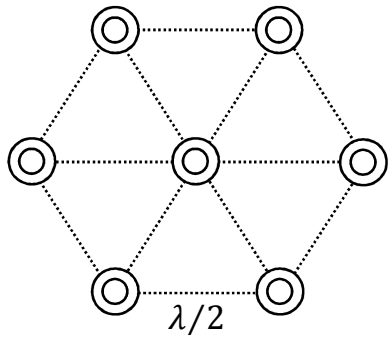

(a)

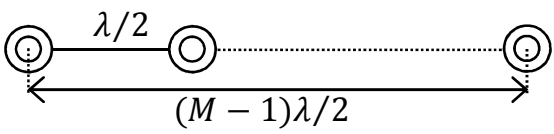

(b)

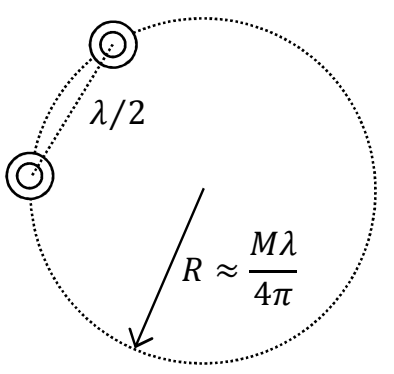

(c)

Figure 1. Simulation configuration (a) UCA-7; (b) ULA; UCA.

Simulated signal has frequency $f_{c}=5 \mathrm{GHz}, \lambda / 2=3 \mathrm{~cm} ; N=10000$ snapshots, and aperture length $L=M \lambda / 2$. DOA of signal is $30^{\circ}$. DOA of interference is $-30^{\circ}$. Noise impacts equally on elements. Monte-Carlo simulation is $Q=200$. When NRMSE is simulated according to SNR, INR $=0 \mathrm{~dB}$, SNR varies in two ranges: high range $[-10 \mathrm{~dB} \div 10 \mathrm{~dB}]$ and low range $[-30 \mathrm{~dB} \div-$ $10 \mathrm{~dB}]$. The purpose of two ranges is to consider the performance when signal donates noise and when signal is weak (for example, the GPS signal is at $-20 \mathrm{~dB}$ ).

Table 1. Simulation parameters.

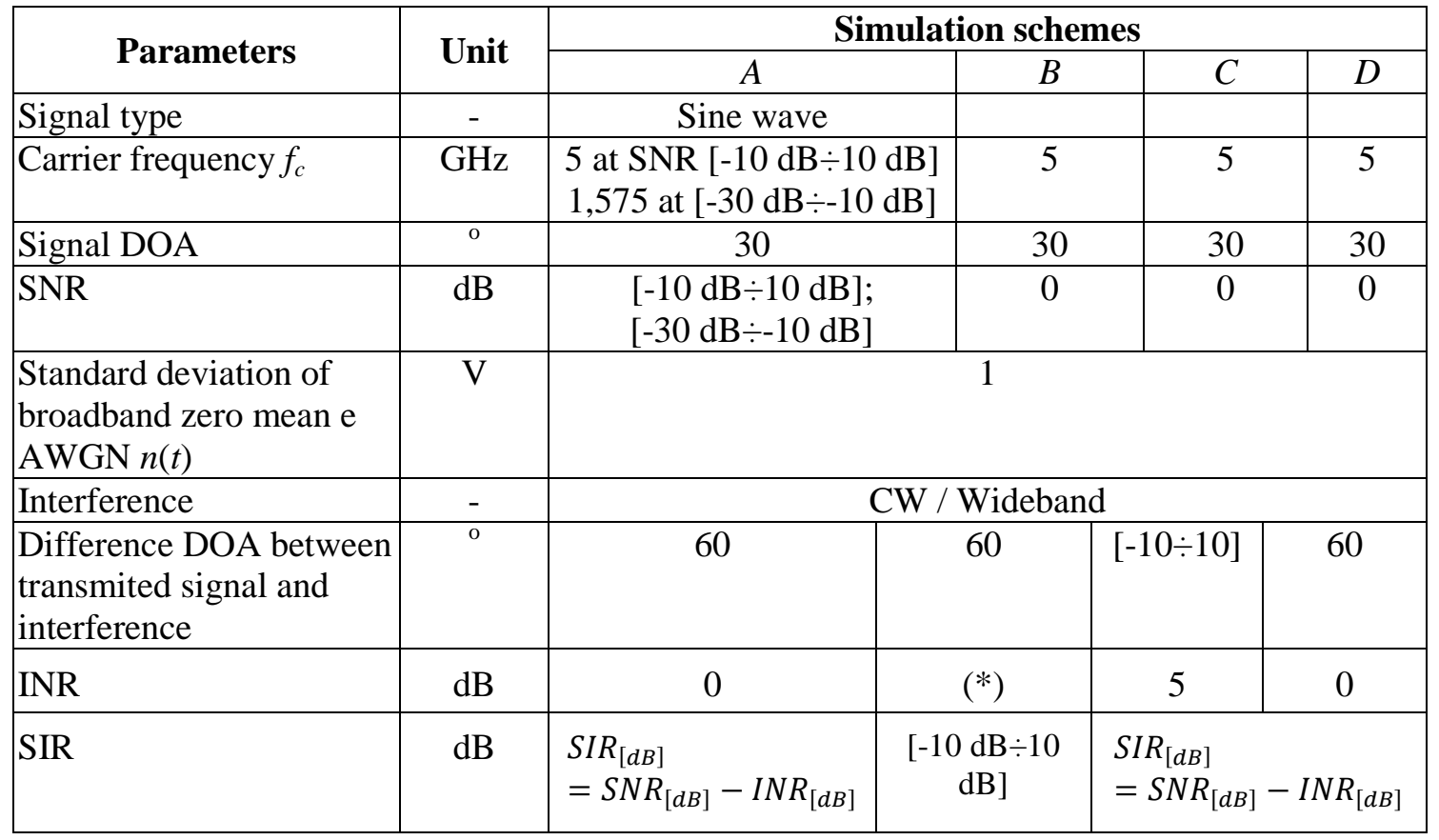




\begin{tabular}{|l|c|c|c|c|c|}
\hline Number of interference & & 1 & 1 & 1 & 1 \\
\hline Array geometry & & ULA/ UCA/ URA & ULA & ULA & ULA \\
\hline Number of antennas $M$ & & $\begin{array}{c}\text { ULA-48; UCA-7; } \\
\text { URA-9 }\end{array}$ & 32 & 32 & $8 \div 168$ \\
\hline Number of samples $N$ & Sample & \multicolumn{3}{|c|}{32 (complex double) } \\
\hline $\begin{array}{l}\text { Sample resolution and } \\
\text { beamforming weight }\end{array}$ & bit & \multicolumn{3}{|c|}{200} \\
\hline Monte-Carlo $Q$ & & \multicolumn{3}{|c|}{$L / 2=M \lambda / 2$} \\
\hline$d$ & & \multicolumn{3}{|c|}{$L / 2000$} \\
\hline$L$ & & \multicolumn{3}{|c|}{} \\
\hline
\end{tabular}

The simulation results are presented in Fig. 2 (a-c) according to SNR ranges. In Fig. 2, we see that when SNR is low, in weak signal range, beamforming based on min-max optimization yields significant result. That is, the system is more robust. But, when signal is good, MVDR provides a better result.

\subsubsection{Signal estimation when SIR varies}

This simulation scheme changes SIR to evaluate the performance under interference.In this case, the DOA of the signal, the DOA of interference are fixed. The carrier frequency is fixed at $5 \mathrm{GHz}$. SNR is $0 \mathrm{~dB}$, note that $I N R_{[d B]}=I S R_{[d B]}+S N R_{[d B]}$. SIR varies in range $[-10 \mathrm{~dB} \div 10$ $\mathrm{dB}$ ]. The simulation results are presented in Fig. 2 (d). We see that the performance of the system according to MVDR and LCMV method give best result, meanwhile the min-max optimization yields bad result when SIR changes.

\subsubsection{Signal estimation when the difference DOA between transmited signal and interference varies}

In this simulation, parameters of signal, array configuration are fixed, the difference DOA between transmited signal and interference varies to consider the dependence of the performance, especially when the DOA of the interference is close to the DOA of the signal. The result shows that the min-max optimization stays the same while MVDR is most affected.

\subsubsection{Signal estimation when the number of antennas changes}

This scheme will change the number of antennas while the rest of parameters stay unchange. SNR $=0 \mathrm{~dB}, \mathrm{INR}=0 \mathrm{~dB}$. Array configuration is ULA, number of antennas is $2^{i}(I=$ $2 \div 7$ ), from 4 to 128 . In this simulation when the number is larger than 72 , the result does not change too much. In order to increase the data speed, we have to combine beamforming method with spatial multiplexing. 


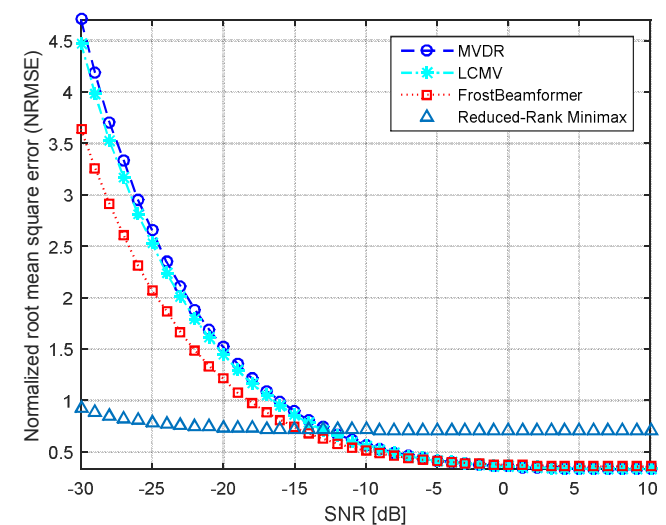

(a)

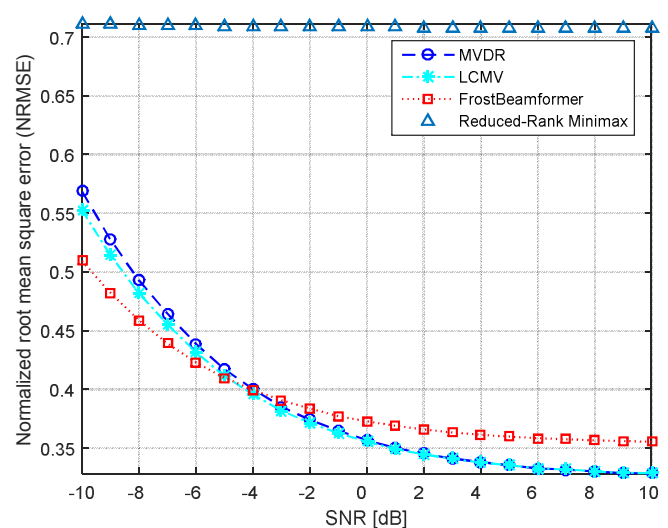

(c)

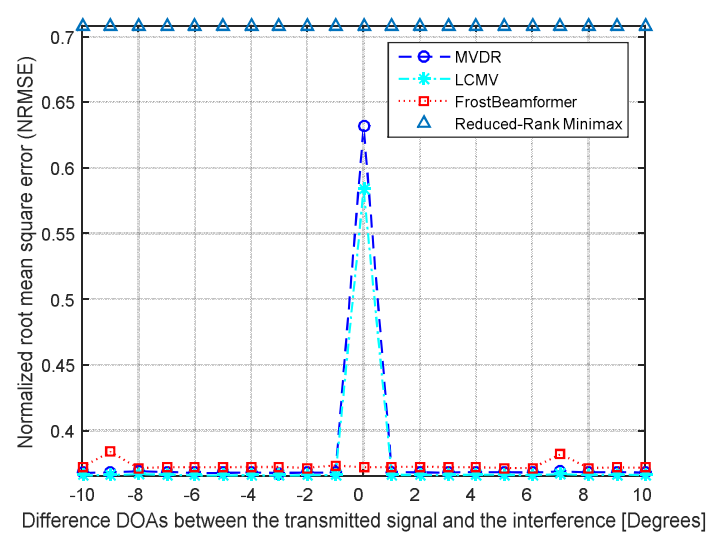

(e)

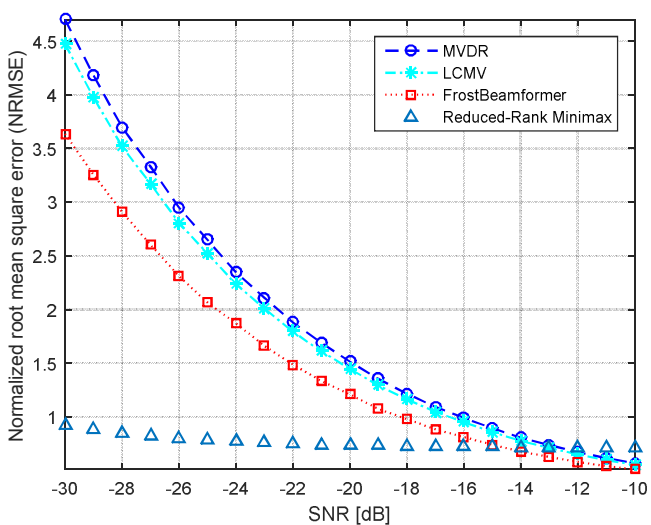

(b)

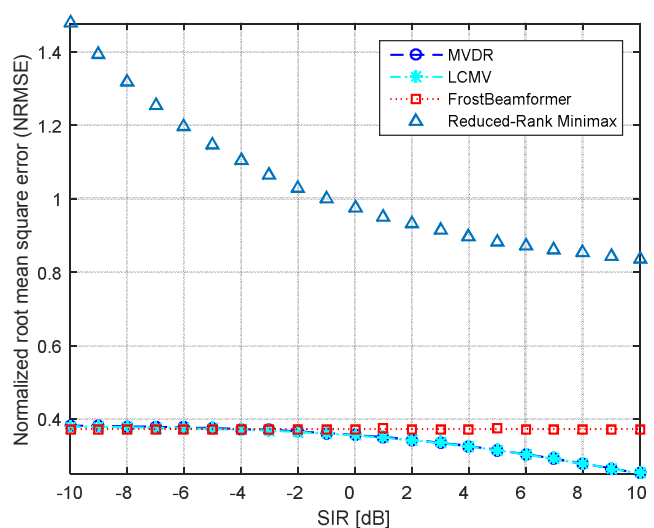

(d)

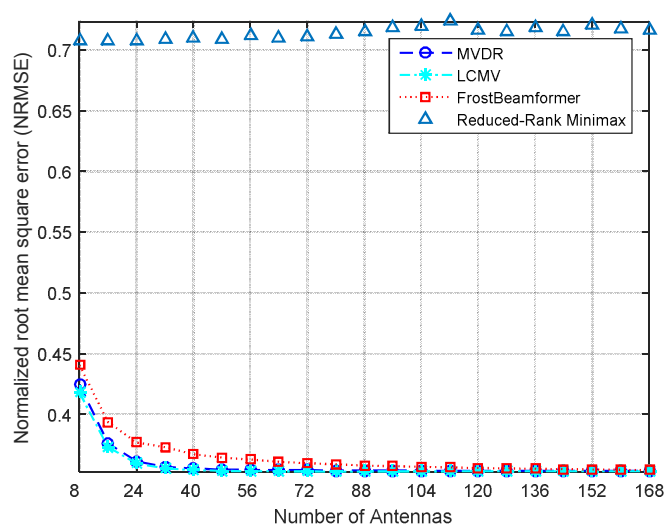

(f)

Figure 2. NRMSE according to SNR (a-c), SIR (d), the difference DOA between transmited signal and interference (e) number of antennas (f).

\section{CONCLUSIONS}

The large scale multiple beamforming system has been analyzed, designed by system theory approach on basis of systematic optimization. Optimization problem to reduced-order 
model in terms of controlability and observability have been developed, using min-max criterion. An important result is when the SNR is low, in weak signal range, min-max optimization yields significant result. The system is more robust. Thus, by systematic approach, the multiple beamforming system can be considered as dymamical system with two parts: unobservative part and uncontrollable part. From that, next research direction in this field is to develop algorithms to strengthen controlability and observability for beamforming system for tracking of moving objects under perturbation and complex environment.

Acknowledgements. The authors would like to thank the Ministry of Science and Technology for the support under the project number NDT.03.ITA/15.

\section{REFERENCES}

1. Magueta R., Castanheira D., Rui S. and Gameiro D. - Non linear space-time equalizer for single-user hybrid mmWave massive MIMO systems, Proceed. 8th International Congress on Ultra Modern Telecommunications and Control Systems and Workshops (ICUMT), 2016.

2. Omar A. and Gustavo L. - Analytical Performance of GNSS Receivers using Interference Mitigation Techniques, IEEE Trans. Aerospace and Electronic Systems 49 (2) (2013) pp. 885-906.

3. Jingwei $\mathrm{Xu}$, Guisheng Liao, Lei Huang, Hing Cheung So - Robust Adaptive Beamforming for Fast-Moving Target Detection With FDA-STAP Radar, IEEE Transactions on Signal Processing 65 (4) (2017) pp. 973 - 984.

4. Vargas D., Gozálvez D., Gómez-Barquero D. and Cardona N. - Multi Antenna Techniques for Digital Video Broadcasting (DVB) Systems, Waves (ISSN 1889-8297) 4 (2012) pp.79-88.

5. Yue Rong, Eldar Y.C. and Gershman A.B. - Performance tradeoffs among beamforming approaches, Proceed. 4th IEEE Workshop on Sensor Array and Multichannel Processing, 2006.

6. Yonina C. Eldar - Minimax MSE Estimation of Deterministic Parameters With Noise Covariance Uncertainties, IEEE Trans. Signal Processing 54 (1) (2006) 138-145.

7. Ming Zhang, Anxue Zhang, Qingqing Yang - Robust Adaptive Beamforming Based on Conjugate Gradient Algorithms, IEEE Transactions on Signal Processing 64 (22) (2016) pp. $6046-6057$.

8. Sergiy A.V., Alex B.G. and Zhi-Quan Luo - Robust Adaptive Beamforming Using WorstCase Performance Optimization: A Solution to the Signal Mismatch Problem, IEEE Trans. Signal Processing 51 (2) (2013) 313-324.

9. Haddad W. M. and Bernstein D. S. - Combine $\mathrm{L}_{2} / \mathrm{H}_{\infty}$ model reduction, Int. J. Contr. 49 (5) (1989) pp.1523-1535.

10. Haddad W. M. and Bernstein D. S. - Robust reduced-order modeling via the optimal projection equations with Petersen-Hollot bounds, IEEE Trans. Auto. Contr. AC-33 (7) (1988) pp. 692-695.

11. Berntein D. S. and Haddad W. M. - LQG controller with an $\mathrm{H}_{\infty}$ performance bound: A Riccati equation approach, IEEE Trans. Auto Contr. AC-34 (4) (1989) pp. 293-305. 
12. San N. N. - On an approach to the estimation state variable descriptive parameters for linear, co models, Optimization 33 (1994) pp.201-217.

13. Isreal A. R. and Greville N. E. - Generalized inverse of matrices: Theory and application, John Wiley \& Sons, NewYork, 1974.

14. San N. N. - State-optimization menthod for order reduction of linear models and of state estimators, Optimization 34 (1995) pp.341-357.

15. Anh N. T. and San N. N. - Controller reduction by state-optimization approach, Optimization 35 (1996) pp.621-640.

16. Aboulnasr H., Matthew W.M., Arash K., Sergiy A.V., Joon-Young P. and Seon-Joo K. Two-dimensional transmit beamforming for MIMO radar with sparse symmetric arrays, Proceed. IEEE Radar Conference, 2013.

17. Giorgio, Giorgi Bienvenido, Jiménez Vicente, Novo - Approximate Karush-Kuhn-Tucker Condition in Multiobjective Optimization, Journal of Optimization Theory and Applications 171 (1) (2016) pp 70-89.

18. Johannes Jahn - Karush-Kuhn-Tucker Conditions in Set Optimization, Journal of Optimization Theory and Applications 172 (3) (2017) pp. 707-725.

19. Pei Chen, Yongjun Zhao, Chengcheng Liu - Robust adaptive beamforming based on sparse reconstruction using a non-convex optimisation algorithm, IET Journals \& Magazines 52 (19) (2016) pp. 1584 - 1586. 